\title{
Causas de Variação de Características de Crescimento de Bovinos Cruzados Canchim x Nelore
}

\author{
Maurício Mello de Alencar ${ }^{1,2}$, Márcia Cristina de Sena Oliveira1, Pedro Franklin Barbosa1
}

RESUMO - O objetivo deste trabalho foi estudar efeitos de fatores genéticos e de meio sobre os pesos ao nascimento (PN), à desmama (PD) e aos 12 (P12), 18 (P18) e 24 (P24) meses de idade e os ganhos de peso do nascimento à desmama (GND) e da desmama aos 12 meses (GD12), dos 12 aos 18 meses (G1218) e dos 18 aos 24 meses (G1824) de idade de animais cruzados Canchim x Nelore, filhos de touros Canchim e vacas Nelores. Os dados foram analisados pelo método dos quadrados mínimos, cujo modelo matemático incluiu os efeitos de ano e mês de nascimento, sexo do bezerro, origem da vaca e idade da vaca ao parto, além dos efeitos aleatórios de pai do bezerro dentro de ano de nascimento. Estimativas de herdabilidade foram obtidas usando-se o componente de variância de pai do bezerro. Ano e mês de nascimento influenciaram todas as características estudadas, com exceção de G1218 e PN, respectivamente. Os animais foram mais pesados e ganharam mais peso, quando a pesagem ocorreu durante ou logo após a estação do verão (janeiro a março). Os machos foram mais pesados que as fêmeas, da desmama aos 24 meses de idade, e ganharam mais peso do nascimento à desmama e dos 18 aos 24 meses de idade. A origem da vaca apresentou efeito apenas sobre PD, GD12 e G1824. A idade da vaca ao parto não influenciou as características estudadas, ao contrário do pai do bezerro, que não influenciou apenas G1824. As estimativas de herdabilidade foram iguais a $0,41 \pm 0,14(\mathrm{PN}), 0,42 \pm 0,15(\mathrm{PD}), 0,53 \pm 0,16(\mathrm{P} 12), 0,46 \pm 0,19(\mathrm{P} 18), 0,63 \pm 0,20(\mathrm{P} 24), 0,37 \pm 0,14(\mathrm{GND}), 0,33 \pm 0,14$ (GD12), 0,34 $\pm 0,17$ (G1218) e $0,15 \pm 0,16$ (G1824).

Palavras-chave: bovinos de corte, fatores de meio, herdabilidade, pesos

\section{Sources of Variation on Growth Traits of Crossbred Canchim x Nellore Cattle}

ABSTRACT - The objective of this work was to study the effect of genetic and non genetic factors on weights at birth (BW), weaning (WW), yearling (YW), 18 months (EW) and 24 months (TW) of age, and weight gains from birth to weaning (GBW), weaning to yearling (GWY), yearling to 18 months (GYE), and 18 to 24 months (GET) of age, of crossbred Canchim x Nellore animals, sired by Canchim ( $5 / 8$ Charolais $+3 / 8 \mathrm{Zebu})$ bulls. The data were studied by the least squares method with a model that included the fixed effects of year and month of birth, sex of calf, cow origin, age of cow at calving, and the random effects of sire within year. Heritabilities were obtained using the sire of calf variance component. Year and month of birth affected all studied traits, except GYE and BW, respectively. The animals were heavier and had higher weight gain when weighed during or just after the summer (from January to March). The males were heavier than the females from weaning to 24 months of age and gained more from birth to weaning, and from 18 to 24 months of age. Cow origin had an effect on WW, GWY, and GET. Age of cow had no effect on the studied traits, while sire of calf did not affect only gain from 18 to 24 months of age. The heritability estimates were $0.41 \pm 0.14(\mathrm{BW}), 0.42 \pm 0.15(\mathrm{WW}), 0.53 \pm 0.16$ (YW), $0.46 \pm 0.19$ (EW), $0.63 \pm 0.20$ (TW), $0.37 \pm 0.14$ (GBW), $0.33 \pm 0.14$ (GWY), $0.34 \pm 0.17$ (GYE) and $0.15 \pm 0.16$ (GET).

Key Words: beef cattle, environmental factors, heritabilities, body weights

\section{Introdução}

O cruzamento entre raças tem sido utilizado no país como meio de aumentar a produtividade dos rebanhos bovinos de corte. ALENCAR (1997), revisando os trabalhos de cruzamento entre raças bovinas no Brasil, observou superioridade dos animais cruzados em relação aos zebus puros para características reprodutivas, de habilidade materna e de crescimento. ALENCAR et al. (1995), MUNIZ et al. (1995) e ALENCAR et al. (1997) verificaram superioridade de animais cruzados Canchim x Nelore em relação aos da raça Nelore para várias características de peso. Para que o cruzamento entre raças possa contribuir efetivamente para o aumento da produtividade dos rebanhos, é importante estudar os efeitos de fatores de meio e genéticos sobre o desempenho dos animais cruzados, visando auxiliar na determinação de práticas de manejo e de critérios de seleção. Fatores de ambiente - ano e mês de nascimento, sexo e idade da vaca ao parto - têm sido apontados como importantes fontes de variação sobre características de crescimento em bovinos de corte (MILAGRES et al., 1985; EUCLIDES FILHO 
et al. 1991; ALENCAR et al., 1993; SOUZA et al., 1994; MASCIOLI et al., 1996b; e ALENCAR et al., 1997). Estimativas de herdabilidade para características de crescimento foram obtidas para animais de raças puras por vários autores no Brasil (ROSA et al., 1986 e 1987; SILVA et al., 1987; NOBRE et al., 1988; ALENCAR et al., 1993; e MASCIOLI et al., 1996a e 1997a,b). Os objetivos deste trabalho foram estudar os efeitos de alguns fatores sobre características de crescimento e obter estimativas de herdabilidade para elas, em animais cruzados Canchim x Nelore.

\section{Material e Métodos}

Foram utilizadas observações dos pesos ao nascimento (PN), à desmama (PD) e aos 12 (P12), 18 (P18) e 24 (P24) meses de idade e dos ganhos de peso do nascimento à desmama (GND), da desmama aos 12 meses (GD12), dos 12 aos 18 meses (G1218) e dos 18 aos 24 meses (G1824) de idade de 629, 601, 577, 394, 429, 601, 577, 391 e 336 animais $1 / 2$ Canchim $+1 / 2$ Nelore, respectivamente. Os animais, filhos de touros da raça Canchim e vacas da raça Nelore, nasceram de 1986 a 1994 e foram criados em regime exclusivo de pastagens dos capins braquiária (Brachiaria decumbens) e andropogon (Andropogon gayanus), recebendo suplementação de minerais, no Centro de Pesquisa de Pecuária do Sudeste (CPPSE) da Empresa Brasileira de Pesquisa Agropecuária (EMBRAPA), situado no município de São Carlos, Estado de São Paulo. As mães dos animais cujos dados foram utilizados neste trabalho eram de cinco origens diferentes, ou seja, provenientes de cinco diferentes rebanhos situados nos Estados de São Paulo e Mato Grosso do Sul.

As pesagens dos animais foram feitas logo após o nascimento (até 24 horas após o nascimento), à desmama (média de 240 dias) e cerca de 30 dias (antes ou após) da data de completarem 12, 18 e 24 meses de idade. Os pesos à desmama, aos 12, 18 e 24 meses de idade foram ajustados para 240,365, $550 \mathrm{e}$ 730 dias, utilizando-se os ganhos diários GND, GD12, G1218 e G1824 e os pesos observados imediatamente anteriores às idades, respectivamente.

Os dados foram analisados pelo método dos quadrados mínimos, cujo modelo matemático foi constituído dos efeitos de ano (1986 a 1994) e mês (maio a dezembro) de nascimento, sexo do bezerro, origem da vaca e idade da vaca ao parto ( $4 \mathrm{a} \geq 11$ anos), além dos efeitos aleatórios de pai do bezerro dentro de ano de nascimento, utilizando-se o procedimento GLM
(SAS, 1996). As estimativas de herdabilidade foram obtidas utilizando-se o componente de variância de pai do bezerro e seus erros-padrão foram obtidos segundo SWIGER et al. (1964).

\section{Resultados e Discussão}

Os resumos das análises de variância são apresentados nas Tabelas 1 e 2 para os pesos e ganhos de peso, respectivamente. Observa-se que o ano de nascimento influenciou significativamente $(\mathrm{P}<0,01)$ todas as características estudadas, com exceção do ganho de peso dos 12 aos 18 meses de idade. Houve tendência de os pesos e ganhos de peso aumentarem com o passar dos anos, com exceção de P12 e GD12, que reduziram significativamente do penúltimo para o último ano de nascimento. Certamente, diferenças na disponibilidade de alimentos, no manejo e genéticas, de ano para ano, foram responsáveis por esses efeitos.

O mês de nascimento apresentou efeito significativo sobre todas as características estudadas, com exceção do peso ao nascimento (Tabelas 1 e 2). As médias estimadas dos pesos e dos ganhos de peso são apresentadas nas Tabelas 3 e 4, respectivamente. Os maiores pesos à desmama e aos 12, 18 e 24 meses de idade ocorreram nos animais nascidos nos períodos de junho a setembro, maio a agosto, agosto a dezembro e maio a agosto, respectivamente. Os maiores ganhos de peso do nascimento à desmama, da desmama aos 12 meses, dos 12 aos 18 meses e dos 18 aos 24 meses ocorreram nos animais nascidos de junho a setembro, em maio, de setembro a dezembro e em maio, respectivamente. Estes maiores pesos e ganhos de peso coincidem com as pesagens ocorridas durante ou após a estação do verão (janeiro a março), ou seja, quando os pastos apresentam elevada disponibilidade de forragem.

O sexo do animal influenciou significativamente os ganhos de peso do nascimento à desmama $(\mathrm{P}<0,01) \mathrm{e}$ dos 18 aos 24 meses $(\mathrm{P}<0,05)$ e os pesos $(\mathrm{P}<0,01)$ da desmama aos 24 meses de idade (Tabelas 1 e 2). Os machos foram de 6,8 a $8,6 \%$ mais pesados que as fêmeas da desmama aos 24 meses de idade (Tabela 5). Os machos ganharam mais peso que as fêmeas do nascimento à desmama $(0,718$ vs. $0,651 \mathrm{~kg} / \mathrm{dia})$ e dos 18 aos 24 meses de idade (0,338 vs. $0,302 \mathrm{~kg} / \mathrm{dia})$. Além das diferenças inerentes aos dois sexos, a superioridade dos machos pode refletir também alguma diferença de manejo que, por ventura, possa ter ocorrido durante o desenvolvimento dos animais, uma vez que o manejo após a desmama, embora exclusivamente a pasto, não foi o mesmo para os dois sexos. 
Rev. bras. zootec.

Tabela 1 - Resumo das análises de variância dos pesos ao nascimento (PN), à desmama (PD) e aos 12 (P12), 18 (P18) e 24 (P24) meses de idade

Table 1 - Analyses of variance of body weights at birth (BW), weaning (WW), and $12(\mathrm{YW}), 18(E W)$, and 24 (TW) months of age

Fonte de variação

Source of variation

\begin{tabular}{|c|c|c|c|c|c|}
\hline \multirow[t]{3}{*}{$\begin{array}{r}\mathrm{gl}^{\mathrm{I}} \\
d f\end{array}$} & \multicolumn{5}{|c|}{$\begin{array}{l}\text { Quadrado médio } \\
\text { Mean square }\end{array}$} \\
\hline & PN & PD & P12 & P18 & P24 \\
\hline & $B W$ & $W W$ & $Y W$ & $E W$ & $T W$ \\
\hline $8 / 8 / 8 / 5 / 6$ & & $4883 * *$ & $3767 * *$ & $4584 * *$ & $30.649^{* *}$ \\
\hline $7 / 7 / 7 / 7 / 7$ & $\begin{array}{c}87 * * \\
35+\end{array}$ & $3251 * *$ & $3451 * *$ & $4205^{* *}$ & $9475^{* *}$ \\
\hline $1 / 1 / 1 / 1 / 1$ & 6 & $34.984^{* *}$ & $35.431 * *$ & $37.665^{* *}$ & $53.563^{* *}$ \\
\hline $4 / 4 / 4 / 4 / 4$ & $40+$ & $1510^{*}$ & 864 & $1661+$ & $2316+$ \\
\hline 7/7/7/7/7 & 26 & 156 & 553 & 763 & 1054 \\
\hline $49 / 49 / 49 / 36 / 37$ & & $981^{* *}$ & $1499^{* *}$ & $1660 * *$ & $2720 * *$ \\
\hline$/ 524 / 500 / 333 / 366$ & $\begin{array}{c}40 * * \\
19\end{array}$ & 485 & 659 & 822 & 1087 \\
\hline & 33 & 50 & 48 & 46 & 51 \\
\hline
\end{tabular}

Ano de nasc.

Year of birth

Mês de nasc.

Month of birth

Sexo

Sex

Origem da vaca

Cow origin

Idade da vaca

Age of cow

Pai (Ano)

Sire (Year)

Resíduo

Error

$\mathrm{R}^{2}(\%)$

$+\mathrm{P}<0,10 ;{ }^{*} \mathrm{P}<0,05 ;{ }^{* *} \mathrm{P}<0,01$

1 Graus de liberdade de PN/PD/P12/P18/P24.

${ }^{1}$ Degrees of freedom of BW/WW/YW/EW/TW.

Tabela 2 - Resumo das análises de variância dos ganhos de peso do nascimento à desmama (GND), da desmama aos 12 meses (GD12), dos 12 aos 18 meses (G1218) e dos 18 aos 24 meses (G1824) de idade

Table 2 - Analyses of variance of body weight gains from birth to weaning (GBW), weaning to 12 months (GWY), 12 to 18 months (GYE), and 18 to 24 months (GET) of age

\begin{tabular}{|c|c|c|c|c|c|}
\hline \multirow[t]{2}{*}{$\begin{array}{l}\text { Fonte de variação } \\
\text { Source of variation }\end{array}$} & \multirow[t]{2}{*}{$\begin{array}{l}\mathrm{gl}^{1} \\
d f\end{array}$} & \multicolumn{4}{|c|}{$\begin{array}{l}\text { Quadrado médio } \\
\text { Mean square }\end{array}$} \\
\hline & & GND & GD12 & G1218 & $\mathrm{G} 1824$ \\
\hline $\begin{array}{l}\text { Ano de nasc. } \\
\text { Year of birth }\end{array}$ & $8 / 8 / 5 / 5$ & $\frac{G B W}{0,086^{* *}}$ & $\frac{G W Y}{0,166^{* *}}$ & $\begin{array}{c}\text { GYE } \\
0,038+\end{array}$ & $\begin{array}{c}G E T \\
0,103^{* *}\end{array}$ \\
\hline $\begin{array}{l}\text { Mês de nasc. } \\
\text { Month of birth }\end{array}$ & $7 / 7 / 7 / 7$ & $0,058 * *$ & $0,239 * *$ & $0,321 * *$ & $0,426 * *$ \\
\hline $\begin{array}{l}\text { Sexo } \\
\text { Sex }\end{array}$ & $1 / 1 / 1 / 1$ & $0,590 * *$ & 0,001 & 0,042 & $0,095^{*}$ \\
\hline $\begin{array}{l}\text { Origem da vaca } \\
\text { Cow origin }\end{array}$ & $4 / 4 / 4 / 4$ & 0,022 & $0,065^{*}$ & 0,030 & $0,051^{*}$ \\
\hline $\begin{array}{l}\text { Idade da vaca } \\
\text { Age of cow }\end{array}$ & 7/7/7/7 & 0,004 & 0,038 & $0,035+$ & 0,029 \\
\hline $\begin{array}{l}\text { Pai (Ano) } \\
\text { Sire (year) }\end{array}$ & $49 / 49 / 36 / 35$ & $0,015^{* *}$ & $0,041^{* *}$ & $0,032 * *$ & 0,026 \\
\hline $\begin{array}{l}\text { Resíduo } \\
\text { Error }\end{array}$ & $524 / 500 / 330 / 276$ & 0,008 & 0,023 & 0,018 & 0,020 \\
\hline $\mathrm{R}^{2}(\%)$ & & 50 & 39 & 55 & 62 \\
\hline
\end{tabular}

$+P<0,10 ;{ }^{*} P<0,05 ;{ }^{* *} P<0,01$.

1 Graus de liberdade de GND/GD12/G1218/G1824.

${ }^{1}$ Degrees of freedom of GBW/GWY/GYE/GET.

Outros autores também verificaram efeitos significativos de ano, mês de nascimento e sexo sobre características de crescimento de animais das raças Nelore e Canchim e cruzados Canchim x Nelore (MILAGRES et al., 1985; ALENCAR et al., 1993; SOUZA et al., 1994; MASCIOLI et al., 1996b; e ALENCAR et al., 1997).
A origem da vaca foi incluída no modelo, pois poderia conter efeitos genéticos, que são transmitidos aos bezerros, e efeitos maternos, que são de origem ambiental para os bezerros. Os efeitos maternos são, por sua vez, de origem genética e de origem do ambiente fornecido às vacas durante seu crescimento. Entretanto, a origem da vaca influenciou $(\mathrm{P}<0,05)$ 
Tabela 3 - Médias $(\mathrm{kg})$ estimadas dos pesos ao nascimento (PN), à desmama (PD) e aos 12 (P12), 18 (P18) e 24 (P24) meses de idade, por mês de nascimento

Table 3 - Least squares means (kg) for body weights at birth (BW), weaning (WW), and 12 (YW), 18 (EW), and 24 (TW) months of age, by month of birth

\begin{tabular}{|c|c|c|c|c|c|}
\hline \multirow{3}{*}{$\begin{array}{l}\text { Mês } \\
\text { Month }\end{array}$} & \multicolumn{4}{|c|}{$\begin{array}{l}\text { Média } \pm \text { erro-padrão } \\
\text { Mean } \pm \text { standard error }\end{array}$} & \multirow[b]{2}{*}{ P24 } \\
\hline & PN & PD & P12 & P18 & \\
\hline & $B W$ & $W W$ & $Y W$ & $E W$ & $T W$ \\
\hline Maio & $30,3 \pm 1,0$ & $186,4 \pm 5,1$ & $231,6 \pm 6,2$ & $257,8 \pm 8,5$ & $370,3 \pm 9,8$ \\
\hline $\begin{array}{l}\text { May } \\
\text { Junho } \\
\text { June }\end{array}$ & $30,5 \pm 0,6$ & $203,1 \pm 3,0$ & $234,4 \pm 3,6$ & $294,7 \pm 8,9$ & $360,7 \pm 7,1$ \\
\hline $\begin{array}{l}\text { Julho } \\
\text { July }\end{array}$ & $29,8 \pm 0,5$ & $205,1 \pm 2,7$ & $228,0 \pm 3,1$ & $295,0 \pm 5,6$ & $358,4 \pm 5,5$ \\
\hline $\begin{array}{l}\text { Agosto } \\
\text { August }\end{array}$ & $31,6 \pm 0,5$ & $208,8 \pm 2,6$ & $223,1 \pm 3,1$ & $305,7 \pm 4,9$ & $348,7 \pm 5,1$ \\
\hline $\begin{array}{l}\text { Setembro } \\
\text { September }\end{array}$ & $31,9 \pm 0,5$ & $201,6 \pm 2,6$ & $216,3 \pm 3,1$ & $309,2 \pm 4,5$ & $337,5 \pm 5,2$ \\
\hline $\begin{array}{l}\text { Outubro } \\
\text { October }\end{array}$ & $31,9 \pm 0,6$ & $197,6 \pm 3,2$ & $210,5 \pm 3,8$ & $304,8 \pm 5,2$ & $340,9 \pm 5,9$ \\
\hline $\begin{array}{l}\text { Novembro } \\
\text { November }\end{array}$ & $31,4 \pm 0,8$ & $186,9 \pm 4,4$ & $215,1 \pm 5,1$ & $303,5 \pm 7,3$ & $323,9 \pm 8,1$ \\
\hline $\begin{array}{l}\text { Dezembro } \\
\text { December }\end{array}$ & $32,6 \pm 1,0$ & $175,0 \pm 5,4$ & $197,8 \pm 6,4$ & $302,7 \pm 7,6$ & $315,6 \pm 9,8$ \\
\hline
\end{tabular}

Tabela 4 - Médias $(\mathrm{kg})$ estimadas dos ganhos de peso do nascimento à desmama (GND), da desmama aos 12 meses (GD12), dos 12 aos 18 meses (G1218) e dos 18 aos 24 meses (G1824) de idade, por mês de nascimento

Table 4 - Least squares means ( $\mathrm{kg}$ ) for body weight gains from birth to weaning (GBW), weaning to 12 months (GWY), 12 to 18 months (GYE), and 18 to 24 months (GET) of age, by month of birth

\begin{tabular}{lcccc}
\hline & \multicolumn{4}{c}{ Média \pm erro-padrão } \\
Mês & GND & GD12 & G1218 & G1824 \\
Month & $G B W$ & $G W Y$ & $G Y E$ & GET \\
\hline $\begin{array}{l}\text { Maio } \\
\begin{array}{l}\text { May } \\
\text { Junho }\end{array}\end{array}$ & $0,649 \pm 0,020$ & $0,380 \pm 0,037$ & $0,229 \pm 0,040$ & $0,650 \pm 0,043$ \\
$\begin{array}{l}\text { June } \\
\text { Julho }\end{array}$ & $0,719 \pm 0,012$ & $0,254 \pm 0,022$ & $0,271 \pm 0,042$ & $0,491 \pm 0,047$ \\
$\begin{array}{l}\text { July } \\
\text { Agosto }\end{array}$ & $0,730 \pm 0,011$ & $0,156 \pm 0,019$ & $0,318 \pm 0,026$ & $0,374 \pm 0,030$ \\
$\begin{array}{l}\text { August } \\
\text { Setembro }\end{array}$ & $0,738 \pm 0,010$ & $0,133 \pm 0,018$ & $0,465 \pm 0,023$ & $0,286 \pm 0,026$ \\
$\begin{array}{l}\text { September } \\
\text { Outubro }\end{array}$ & $0,707 \pm 0,010$ & $0,122 \pm 0,019$ & $0,525 \pm 0,021$ & $0,200 \pm 0,025$ \\
$\begin{array}{l}\text { October } \\
\text { Novembro }\end{array}$ & $0,690 \pm 0,013$ & $0,129 \pm 0,023$ & $0,546 \pm 0,025$ & $0,260 \pm 0,029$ \\
$\begin{array}{l}\text { November } \\
\text { Dezembro }\end{array}$ & $0,648 \pm 0,017$ & $0,241 \pm 0,030$ & $0,529 \pm 0,034$ & $0,145 \pm 0,046$ \\
December & $0,595 \pm 0,022$ & $0,273 \pm 0,038$ & $0,542 \pm 0,036$ & $0,154 \pm 0,044$ \\
\hline
\end{tabular}

apenas o peso à desmama e os ganhos de peso da desmama aos 12 meses e dos 18 aos 24 meses de idade (Tabelas 1 e 2). Para os pesos ao nascimento e aos 18 e 24 meses de idade, o efeito da origem da vaca foi significativo apenas no nível de $10 \%$ de probabilidade.

A idade da vaca ao parto não apresentou efeito significativo sobre nenhuma das características estudadas (Tabelas 1 e 2), o que não está de acordo, pelo menos para o crescimento até a desmama, com a maioria dos trabalhos publicados na literatura científica (MILAGRES et al., 1985; EUCLIDES FILHO et al., 1991; SOUZA et al., 1994; e MASCIOLI et al., 1996b).

O pai do animal apresentou efeito significativo $(\mathrm{P}<0,01)$ sobre todas as características estudadas, com exceção do ganho de peso dos 18 aos 24 meses de idade. Este é um efeito de origem genética e os resultados obtidos estão de acordo com os verificados por ALENCAR et al. (1993) e MASCIOLI et al. 
Rev. bras. zootec.

Tabela 5 - Médias $(\mathrm{kg})$ estimadas dos pesos ao nascimento $(\mathrm{PN})$, à desmama $(\mathrm{PD})$ e aos $12(\mathrm{P} 12), 18$ (P18) e 24 (P24) meses de idade, por sexo

Table 5 - Least squares means (kg) for body weights at birth (BW), weaning (WW), and 12 (YW), 18 (EW), and 24 (TW) months of age, by sex

\begin{tabular}{|c|c|c|c|c|c|}
\hline \multirow{3}{*}{$\begin{array}{l}\text { Variável } \\
\text { Variable }\end{array}$} & \multicolumn{5}{|c|}{$\begin{array}{l}\text { Média } \pm \text { erro-padrão } \\
\text { Mean } \pm \text { standard error }\end{array}$} \\
\hline & PN & PD & P12 & P18 & P24 \\
\hline & $B W$ & $W W$ & $Y W$ & $E W$ & $T W$ \\
\hline \multicolumn{6}{|l|}{ Sexo } \\
\hline Sex & & & & & \\
\hline $\begin{array}{l}\text { Macho } \\
\text { Male }\end{array}$ & $31,4 \pm 0,3$ & $203,7 \pm 1,7$ & $227,9 \pm 2,1$ & $307,2 \pm 3,8$ & $356,0 \pm 4,0$ \\
\hline $\begin{array}{l}\text { Fêmea } \\
\text { Female }\end{array}$ & $31,2 \pm 0,3$ & $187,5 \pm 1,7$ & $211,3 \pm 2,1$ & $286,1 \pm 3,6$ & $333,1 \pm 3,8$ \\
\hline $\begin{array}{l}\text { Geral } \\
\text { Overall }\end{array}$ & $31,3 \pm 0,2$ & $195,6 \pm 0,9$ & $219,6 \pm 1,0$ & $296,7 \pm 1,4$ & $344,5 \pm 1,6$ \\
\hline
\end{tabular}

(1996b e 1997b) na raça Canchim.

As médias estimadas ( \pm erros-padrão) obtidas foram iguais a 31,3 $\pm 0,2(\mathrm{PN}), 195,6 \pm 0,9(\mathrm{PD})$, $219,6 \pm 1,0(\mathrm{P} 12), 296,7 \pm 1,4(\mathrm{P} 18)$ e $344,5 \pm 1,6$ (P24) $\mathrm{kg}$ e 0,684 $\pm 0,003$ (GND), 0,211 $\pm 0,006$ (GD12), 0,428 $\pm 0,007$ (G1218) e $0,320 \pm 0,008$ (G1824) kg/dia (Tabela 5).

As estimativas de herdabilidade foram iguais a $0,41 \pm 0,14(\mathrm{PN}), 0,42 \pm 0,15(\mathrm{PD}), 0,53 \pm 0,16$ (P12), 0,46 \pm 0,19 (P18), 0,63 $\pm 0,20$ (P24), $0,37 \pm 0,14$ (GND), 0,33 $\pm 0,14$ (GD12), $0,34 \pm 0,17$ (G1218) e $0,15 \pm 0,16$ (G1824). As estimativas para os pesos são altas, indicando que a seleção para estas características deve resultar em progresso genético. Estes valores diferem dos obtidos por ROSA et al. (1986 e 1987), SILVA et al. (1987) e NOBRE et al. (1988), para a raça Nelore, e ALENCAR et al. (1993) e MASCIOLI et al. (1996a e 1997a,b), para a raça Canchim, que utilizaram a mesma metodologia usada neste trabalho. Fatores como ambiente, amostra de dados, modelo matemático e metodologia, normalmente, influem na magnitude das herdabilidades (MASCIOLI et al., 1996a). Além desses fatores, segundo BENNETT e GREGORY (1996), a variância genética pode variar entre populações de bovinos por outras razões, entre elas, as diferenças nas freqüências gênicas e as relações funcionais como, por exemplo, componentes de escala com o peso do animal e restrições de crescimento pela alimentação limitada pela mãe. Dessa maneira, esperam-se diferenças nas herdabilidades obtidas para diferentes rebanhos dentro de um mesmo grupo genético e para diferentes grupos genéticos.

\section{Conclusões}

Os fatores de meio ano e mês de nascimento e sexo do bezerro foram importantes fontes de variação e devem ser considerados por ocasião da obtenção de parâmetros genéticos para pesos e ganhos de peso de bovinos cruzados Canchim x Nelore. A seleção para os pesos do nascimento aos 24 meses de idade deve resultar em progresso genético.

\section{Referências Bibliográficas}

ALENCAR, M.M. Los cruzamientos para la producción de carne bovina. In: CONGRESO INTERNACIONAL DE TRANSFERENCIA DE TECNOLÓGICA AGROPECUARIA, 5, 1997, Asunción. Anais... Asunción: CEA, 1997. p.111-122.

ALENCAR, M.M., BARBOSA, P.F., BARBOSA, R.T. et al. 1993. Parâmetros genéticos para peso e circunferência escrotal em touros da raça Canchim. R. Soc. Bras. Zootec., 22(4):572-583.

ALENCAR, M.M., BARBOSA, P.F., TULLIO, R.R. et al. 1995. Peso à desmama de bezerros da raça Nelore e cruzados Canchim x Nelore e Marchigiana x Nelore. R. Soc. Bras. Zootec., 24(6):917-925.

ALENCAR, M.M., TREMATORE, R.L., BARBOSA, P.F. et al. 1997. Desempenho de bezerros filhos de touros das raças Nelore e Canchim e cruzados $\left(\mathrm{F}_{1}\right)$ Charolês x Nelore e Piemontês x Nelore. R. Bras. Zootec., 26(3):461-466.

BENNETT, G.L., GREGORY, K.E. 1996. Genetic (co)variances among birth weight, 200-day weight, and postweaning gain in composites and parental breeds of beef cattle. J. Anim. Sci., 73(11):2598-2611.

EUCLIDES FILHO, K., NOBRE, P.R.C., ROSA, A.N. 1991. Idade da vaca e suas inter-relações com a fazenda, reprodutor e sexo do bezerro. R. Soc. Bras. Zootec., 20(1):40-46.

MASCIOLI, A.S., ALENCAR, M.M., BARBOSA, P.F. et al. 1996a. Estimativas de parâmetros genéticos e proposição de critérios de seleção para pesos na raça Canchim. R. Soc. Bras. Zootec., 25(1):72-82.

MASCIOLI, A.S., ALENCAR, M.M., BARBOSA, P.F. et al. 1996b. Influência de fatores de meio sobre pesos de animais 
da raça Canchim. R. Soc. Bras. Zootec., 25(5):853-865.

MASCIOLI, A.S., ALENCAR, M.M., BARBOSA, P.F. Estimativas de parâmetros genéticos e fenotípicos para ganhos de peso na raça Canchim. In: REUNIÃO ANUAL DA SOCIEDADE BRASILEIRA DE ZOOTECNIA, 34, 1997, Juiz de Fora. Anais... Juiz de Fora: SBZ, 1997a, p. 233-234.

MASCIOLI, A.S., PAZ, C.P., FARO, L. et al. 1997b. Estimativas de parâmetros genéticos e fenotípicos para características de crescimento até a desmama em bovinos da raça Canchim. R. Bras. Zootec., 26(4):709-713.

MILAGRES, J.C., SILVA, L.O.C., NOBRE, P.R.C. et al. 1985. Influência de fatores de meio e herança sobre pesos de animais da raça Nelore no Estado de Minas gerais. R. Soc. Bras. Zootec., 14(4):463-484.

MUNIZ, C.A.S.D., QUEIROZ, S.A., ALBUQUERQUE, L.G. Avaliação de características de crescimento de animais Nelore e seus cruzados. In: REUNIÃO ANUAL DA SOCIEDADE BRASILEIRA DE ZOOTECNIA, 32, 1995, Brasília. Anais... Brasília: SBZ, 1995, p.645-646.

NOBRE, P.R.C., ROSA, A.N., EUCLIDES FILHO, K. 1988. Interações reprodutor $\mathrm{x}$ estação de nascimento e reprodutor $\mathrm{x}$ fazenda sobre o crescimento de bezerros Nelore. $R$. Soc. Bras. Zootec., 17(2):120-131.

ROSA, A. N., NOBRE, P.R.C., EUCLIDES FILHO, K. Desenvolvimento ponderal de animais da raça Nelore variedade mocha: parâmetros genéticos e de ambiente. In: REUNIÃO ANUAL DA SOCIEDADE BRASILEIRA DE ZOOTECNIA, 24, 1987, Brasília. Anais... Brasília: SBZ, 1987, p.286.
ROSA, A. N., SILVA, L.O.C., NOBRE, P.R.C. 1986. Avaliação do desempenho de animais Nelore em controle do desenvolvimento ponderal no Estado do Mato Grosso do Sul-Brasil. R. Soc. Bras. Zootec., 15(6):515-532.

SAS statistical analysis systems user's guide: Stat, Version 6.12 ed. Cary: SAS Institute, 1996.

SILVA, L.O.C., ROSA, A.N., NOBRE, P.R.C. et al. 1987. Análise de pesos de bovinos Nelore criados a pasto no Estado de São Paulo, Brasil. Pesq. Agropec. Bras., 22(11/12):12451256.

SOUZA, J.C., BRULÉ, A.V., FERRAZ FILHO, P.B. et al. 1994. Repetibilidade dos pesos e ganho de peso do nascimento à desmama de bovinos da raça Nelore. R. Soc. Bras. Zootec., 23(1):133-138.

SWIGER, L.A., HARVEY, W.R., EVERSON, D.U. et al. 1964. The variance of intraclass correlation involving groups with one observation. Biometrics, 20:818-826.

Recebido em: 12/02/98

Aceito em: 15/03/99 